\title{
A wind turbine emulator for generator control algorithm development
}

\author{
Luca Peretti*, Ville Särkimäki*, Jan Faber ${ }^{\dagger}$ \\ *ABB Corporate Research, Department of Power Technologies \\ Forskargränd 7, 72178 Västerås, Sweden \\ Email: [luca.peretti,ville.sarkimaki]@se.abb.com \\ ${ }^{\dagger}$ University of Žilina, Department of Power Electrical Systems \\ Univerzitná 1, 01026 Žilina, Slovak Republic \\ Email: faberjan@post.sk
}

\begin{abstract}
The paper describes an emulator for wind turbines which aims to be a base for the development of control algorithms for wind turbine generators. The emulator is physically composed by two servomotors in a back-to-back configuration, each of them controlled by a 4-kW power electronics converter. A FPGAbased control board is employed to model the effects of the wind, both in terms of turbulence and harmonics, and to emulate the mechanical characteristics of a wind turbine drive train. The models generate torque reference signals for the torque control of one of the two machines, which acts as a wind turbine. The second machine is used as a generator, controlled by the same FPGA-based board.
\end{abstract}

The specific tasks of a wind turbine emulator are presented, describing in detail how they could be replicated on a small-scale test bench. The features of the actual emulator shown in the paper are discussed. Experimental results are presented, showing the benefits of a small-scale system for algorithm development.

Index Terms-Wind turbine, wind models, small-scale prototype, FPGA implementation

\section{INTRODUCTION}

Wind energy and wind turbines are always associated with the concept of renewable energy and its applications. As a matter of fact, however, wind turbines can be considered as one of the most challenging mechatronic systems, merging very different disciplines of engineering.

Within this frame, the design of wind turbines, and in particular of wind turbine components (such as generators, converters or gearboxes), should not forget about the peculiarity of the application. As an example, torque cycles and wind speed variations should be considered when designing a proper generator for wind turbines - and the power converter control design should not forget its basic role in the power generation towards the grid.

Challenges in the field of wind energy are manifold, and the bigger is the turbine rated power, the more difficult it becomes to test the wind turbine subsystems on specific field conditions prior delivery and commissioning. Therefore, inhouse testing with smaller-scale prototypes is gaining more and more interest. Especially when dealing with the test of control algorithms for wind turbine generators, it is even more essential that the experiments represent realistic load cycles on the electrical machines, as the power electronics converter deals with the system as a whole with its electrical, electronic and mechanical aspects.

Examples of small-scale prototypes for wind energy applications have been presented in the past literature. In the vast majority of the cases, they are based on models of single wind turbines that have also been developed throughout the years. As a matter of fact, many research efforts in the modelling area have been directed to a better understanding of the wind behaviour and of the effects of big inertias on the mechanical drive train. Interesting attempts, as for example in [1], tried to link the models with experimental measurements. A fairly detailed model developed for power system studies, but which also comprises wind behaviour and a generator control model, is shown in [2]. More detailed wind models, accounting both for turbulence effects and for tower shadow and wind shear effects, are described in [3] and [4]. As the specific wind modelling is a research branch which is not directly linked to the electrical drives scenario, the Reader is invited to refer to the cited documentation for further details on the topic.

The implementation of these models in experimental test benches is not always immediate. Interesting attempts are described, for example, in [5], [6], [7] and [8], where the last is probably the most detailed work. All the implementation have been focused on back-to-back configuration of electrical machines, where one of the two machines acts as a wind turbine. The tower shadow and wind shear effects are also implemented with different solutions, where [8] is the most analytical in this sense, based on the work of the same Authors in [9]. However, no clear considerations has been made on the emulation of the pitch control, and also turbulence effects does not always have a clear explanation both in theory and in its implementation.

This paper is organised as follows. Sect. II gives an overview of the proposed wind turbine emulator, by describing the real system and dividing the emulation problem in different, smaller tasks. Sect. III describes in detail the emulator of the wind pattern, by analysing the implementation of some wind models taken from the literature which encompass both turbulence and specific harmonics. Sect. IV discusses briefly the emulation of mechanical characteristics which are not present in the mechanical system of the small-scale proto- 
type, as for example the reproduction of resonances. Sect. $\mathrm{V}$ describes the actual small-scale prototype and its features, showing some experimental results. Some conclusive remarks ends the paper.

\section{A SYSTEM OVERVIEW}

A system overview is necessary to understand the needs of the wind turbine emulator. Fig. 1 shows a general scheme of a wind turbine system, taken and modified from [10].

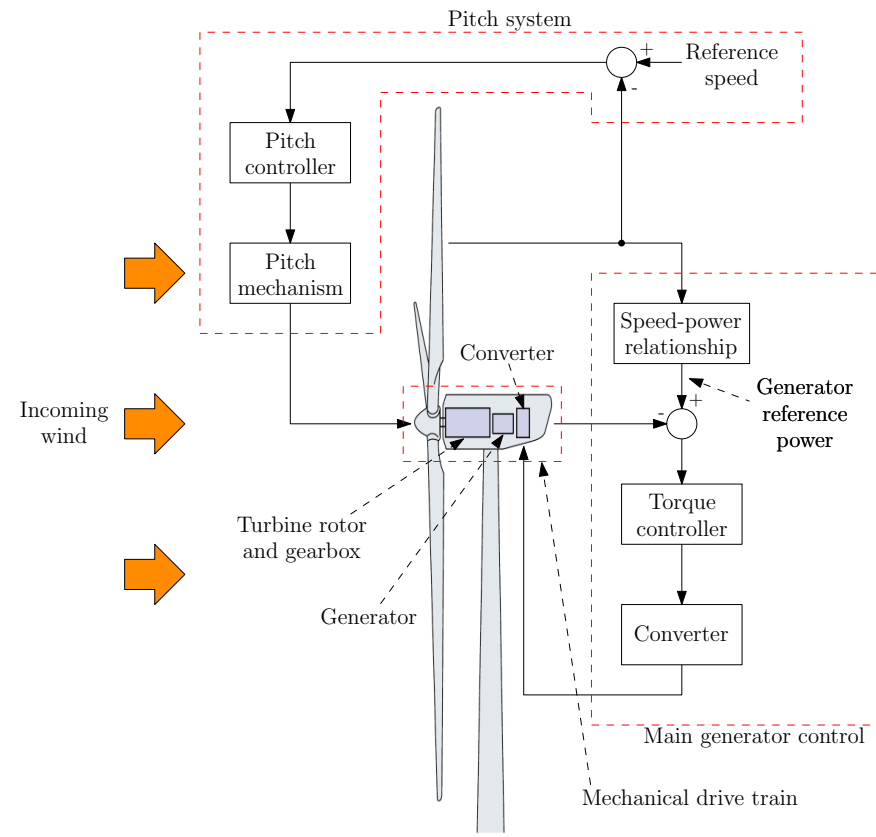

Fig. 1. Schematic of a wind turbine system (modified from [10]).

The incoming wind on the left side of Fig. 1 represents the main source of (mechanical) torque, and therefore must be properly modelled. Effects like deviations from the main average wind speed, caused by turbulence, should be taken into account, and other secondary effects due to the aerodynamic interaction of the wind flow with the blades and the tower should be also considered. In particular, tower shadow and wind shear effects should be modelled: tower shadow is the effect of the wind flow deviation around the tower of the wind turbine, which reduces the actual torque exerted on the blades when each of the blades passes through the tower [1], [9]. Wind shear is the effect of the wind flow distribution from the ground to the higher point reached by one blade in perfect vertical position, which causes differences in torque exerted on the blades depending on the actual vertical position of the blades [1], [9]. Both effects result in torque harmonics which are equal to the number of blades - in conventional three-blade wind turbines, the disturbance is a third harmonic of the main rotating frequency.

The dashed box indicated as "main generator control" is the one referring to the control algorithm for power generation. Being not the main focus of this work, its implementation will not be described hereafter.
The dashed box indicated as "pitch system" represents the actuators and the control systems performing blade pitch control, with primary goal to keep the turbine rotor speed within the rated limits and compensate, to some extent, the third-harmonic disturbances mentioned above. In general terms, the pitch system can be also considered as a sort of speed controller on the turbine side, with a lower disturbance rejection with respect to the conventional speed regulators in industrial electrical drives. Moreover, there is a main difference between a pitch system and a conventional speed controller. Any torque disturbance (harmonic) not originated by the wind flow pattern and appearing on the mechanical drive train is not compensated by the pitch regulator, which can only turn the blades in order to stabilize the system speed. On the contrary, conventional industrial drives employs a speed regulation where disturbances can be mitigated to some extent, depending on the bandwidth and performances of the speed regulator.

However, an approximation of a pitch system can be obtained by a keen implementation of the cascaded speed and torque regulation in the industrial drive used for the smallscale test bench. The proposed solution is shown in Fig. 2.

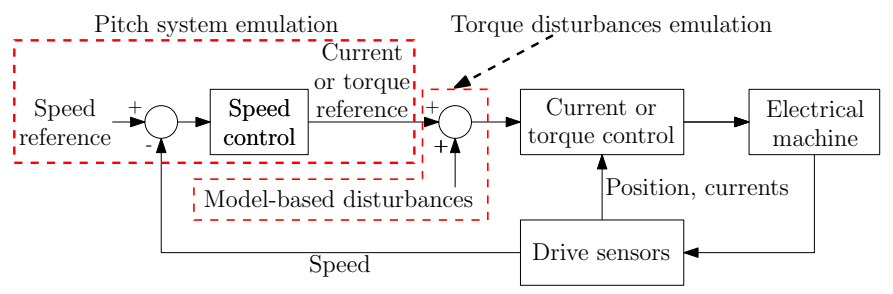

Fig. 2. Proposed implementation of the pitch system and the wind pattern.

Fig. 2 depicts the general scheme of a cascade speed and current/torque controller. The pitch system is emulated by means of the speed controller, by keeping in mind that the parameters of the speed regulator (proportional and integral terms) must be reduced in order not to compensate torque disturbances. In other words, the bandwidth of the speed regulator must be released and kept at low values. In this way, the speed controller will maintain a rough constant speed, allowing the superimposition of torque disturbances coming from different sources.

The easiest way to introduce the other torque disturbances in the emulator is also shown in Fig. 2. An intermediate signal positioned in the middle of the speed-current control chain can bring model-based current or torque references which, in this case, could represent the wind turbulence not compensated by the pitch system or the mechanical drive train harmonics originated by the generator or the grid. As a matter of fact, the exploitation of the speed controller as a simplified pitch system also leads to enhanced safety, as torque disturbances are not able to drift the speed of the test bench to excessive values.

The last dashed block in Fig. 1 refers to the "Mechanical drive train" issue. The mechanical shaft in the experimental test bench is inherently shorter and most probably more stiff 
than the mechanical shaft on an actual wind turbine. Moreover, as addressed in other works like [6] and [8], the inertia of a wind turbine is much higher than the generator one, as it is not in a small-scale test bench. Therefore, proper actions should be taken in order to take into account these effects.

\section{THE WIND PATTERN EMULATOR}

In order to implement a fairly detailed model of the wind behaviour, the works carried out in [3] and [4] have been chosen. In those papers, the wind models are based on the Kaimal spectra, and an equivalent wind speed is calculated as the average of the punctual wind speed over the rotor of the wind turbine. The Authors managed to take into account the tower shadow, the wind shear and the rotational turbulences directly in the calculation of the equivalent wind speed. Thus, the relation between the wind speed and the torque exerted on the shaft can be easily implemented with one algebraic relation, as it will contain the harmonics coming from the wind. A general schematic of the wind model is shown in Fig. 3.

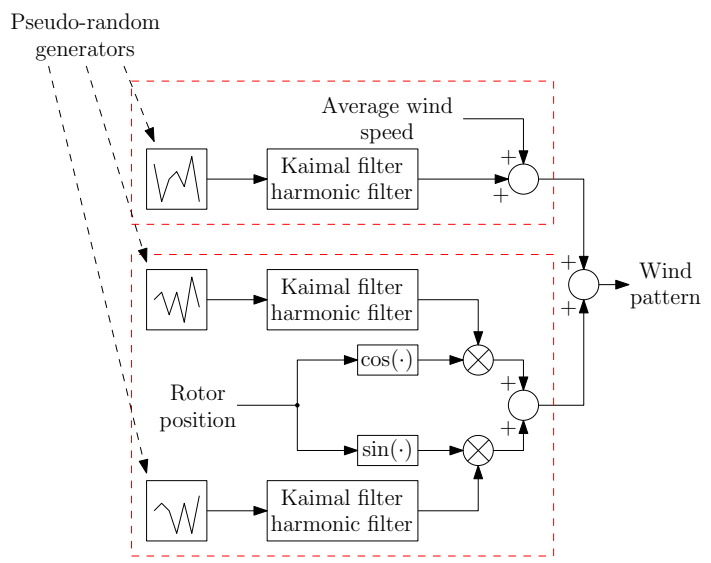

Fig. 3. General scheme of a wind pattern simulator.

Such models, while giving an easy handling of both turbulence and harmonic effects, were not originally developed for experiments on a test bench. Therefore, precautions should be taken in their implementation, in order to preserve their characteristics.

One of the most important components in the wind model is the random generator, which must be a normally-distributed white-noise generator. Almost all dynamic simulation programs have a built-in library for pseudo-random number generation, but there is a clear issue in implementing such generation within a FPGA environment. A solution that encompass both a flat power spectral density and a simple and effective solution for FPGA implementation has been found in [11]. The work of [11] presents a white-noise generator composed by the combination of three smaller pseudo-random number generators. However, just one of these smaller pseudorandom number generators could be sufficient for the purposes of the wind turbine emulator.
In particular, the chosen pseudo-random number generator is based on the expressions (1), where $\gg$ is a bitwise shift operator and \& is a bitwise AND operator.

$$
\begin{aligned}
& S_{k}=30903 x_{k-1}+\left(S_{k-1} \gg 16\right) \\
& x_{k}=S_{k} \& 65535
\end{aligned}
$$

Its straightforward implementation in a FPGA environment is shown in Fig. 4.

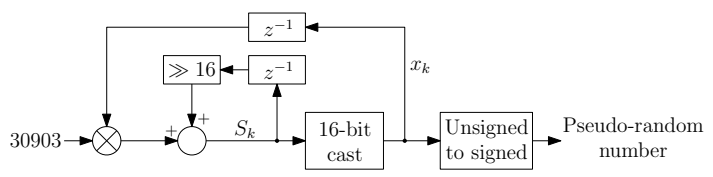

Fig. 4. Implementation of the pseudo-random number generator.

The sequence in (1), which gives different results depending on the choice of the initial seeds $x_{k-1}$ and $S_{k-1}$ (which must be non-zero), is capable of producing a sequence of 16-bit pseudo-random integer numbers with a period greater than $2^{29}$ cycles [11]. A proof of the white-noise pseudo-random number generator was performed, calculating a sequence of 300000 16-bit samples and storing the results in different bins of size 500. Fig. 5 shows that the sequence (1) equally distributes its output values over the whole range from 0 to 65535, proving its white-noise features.

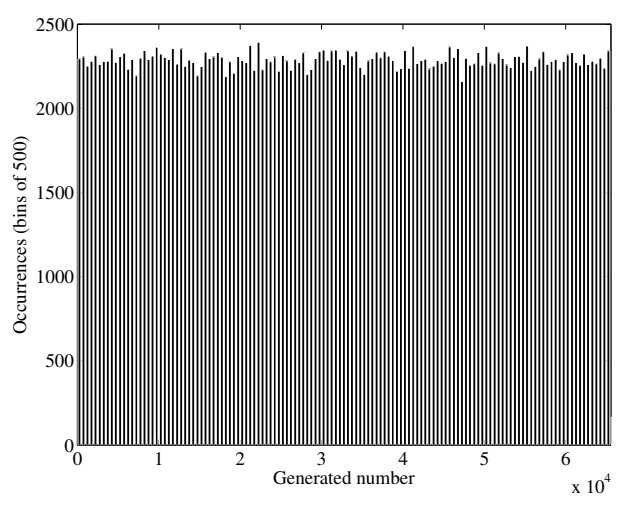

Fig. 5. Proof of the white-noise randomness of the sequence (1).

For digital implementation issues related to the Kaimal filters shown in Fig. 3, the sampling frequency of the filters and the random generators has been set to $0,16 \mathrm{~s}$, which is anyway higher than the normal turbulence activity of the wind. In this condition, the pseudo-random number sequence will repeat itself after more than 23000 hours.

Three simulations of the wind model of Fig. 3, before the FPGA implementation, are shown in Fig. 6, Fig. 7 and Fig. 8. Fig. 6 reports the wind pattern dynamics in the time domain over a range of $500 \mathrm{~s}$. The turbulence behaviour is clearly noticeable. Fig. 7 is a zoom of the previous figure, where the presence of the third harmonics is noticeable. Fig. 8 is a fastFourier transformation of the time sequence, confirming the presence of a third harmonic around $6 \mathrm{~Hz}$, considering that the main rotating frequency was set to $2 \mathrm{~Hz}$. It is worth to 
note that the wind pattern values are 16-bit digital integers, as the whole wind turbine system is implemented in digital domain with fixed-point variables.

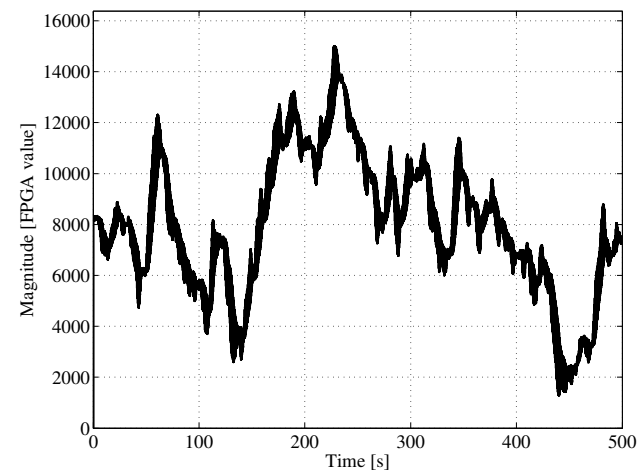

Fig. 6. Simulated wind model dynamics.

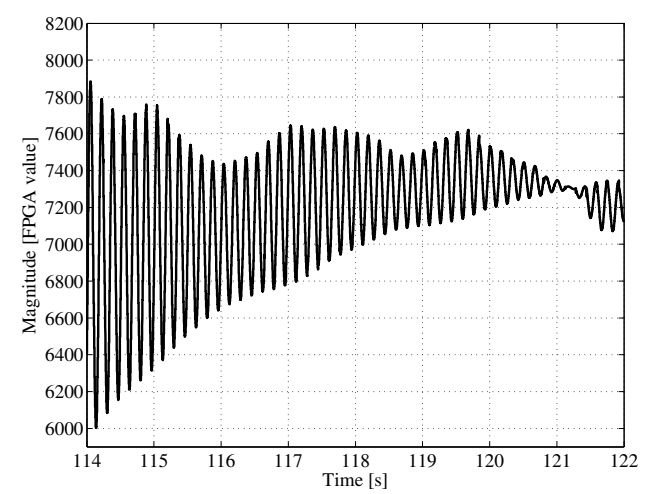

Fig. 7. Simulated wind model dynamics: zoom over a limited time range.

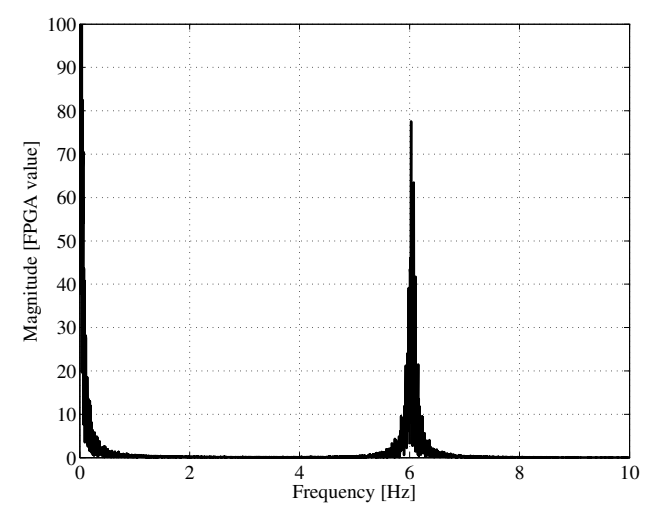

Fig. 8. Simulated wind model dynamics: FFT of the time sequence.

\section{THE MECHANICAL DRIVE TRAIN EMULATOR}

As a matter of fact, the connection of the rotor of a wind turbine with an electrical machine acting as generator represents a mechanical system with very specific characteristics. First of all, the inertias of the system (that is, the one of the rotor and the one of the generator) are very different to each other, with that of the rotor much larger than that of the generator. Moreover, the presence of gearboxes and/or long mechanical shafts leads to the presence of low-frequency resonances which should be taken into account within a wind turbine emulator.

As regards to the first issue, previous works have already recognised the need of emulating an increased inertia ([7], [8]). The mathematical formulation has been developed by considering a rigid system for the mechanical shaft of the test bench, without any viscous friction nor resonances. The mechanical drive train system is also considered as a rigid system without friction, where the inertia of the rotor is larger than that of the generator.

In this work, while the mechanical connection of the test bench is still considered rigid, friction is taken into account. More important, the drive train system of the wind turbine is considered with its resonances and frictions. Such step requires a more general approach for the emulation of mechanical drive trains with respect to that of [7] and [8]. The details on the theoretical developments on this issue are reported in [12], and are only briefly recalled hereafter.

Fig. 9 shows an active load emulation diagram performed through an a torque-controlled drive $C_{1}$ coupled with another electrical drive $C_{2}$. Control references for $C_{2}$ are given through the scheme in the dashed box. $M_{1}$ and $M_{2}$ represent the actual transfer functions between the torque generated by the two movers and the mechanical speed, while $M_{1, e m}$ and $M_{2, e m}$ represent the transfer functions that the drive emulator should reproduce. $\tau_{1}^{*}$ and $\tau_{2}^{*}$ represent the torque references generated for the torque control.

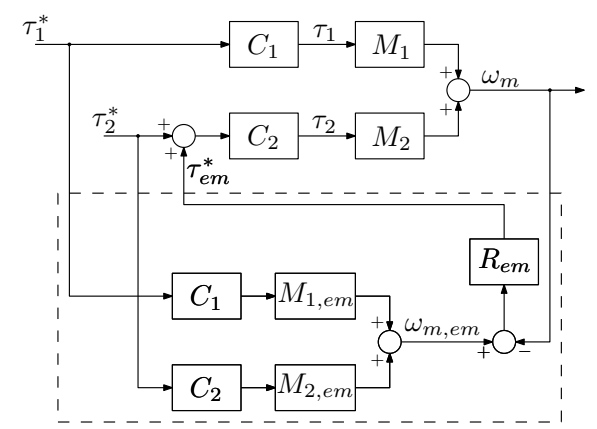

Fig. 9. Block diagram of an active load emulation with an electrical drive.

The transfer functions of the scheme are the following [12]:

$$
\begin{aligned}
\omega_{m}= & \frac{M_{1}+M_{2} C_{2} R_{e m} M_{1, e m}}{1+M_{2} C_{2} R_{e m}} \tau_{1}+ \\
& \frac{M_{2}\left(1+R_{e m} M_{2, e m} C_{2}\right)}{1+M_{2} C_{2} R_{e m}} \tau_{2}
\end{aligned}
$$

Therefore, the conditions for tuning the regulator $R_{e m}$ are:

$$
\begin{aligned}
\omega_{m} \cong & M_{1, e m} \tau_{1}+M_{2, e m} \tau_{2} \\
& \text { for } M_{1, e m} M_{2} C_{2} R_{e m} \gg M_{1} \text { and } M_{2} C_{2} R_{e m} \gg 1 \\
& \text { and } M_{2, e m} C_{2} R_{e m} \gg 1
\end{aligned}
$$


The same expressions can be used to determine the rating of the machine and the power electronics converter for the load emulation. For more details, the Reader is redirected to [12]. It is anyway worth to underline that such emulation considers the drive train as a fixed mechanical system. According to [13], this is only an approximation of reality, as resonances in wind turbine drive trains change depending on the load conditions.

\section{EXPERIMENTAL TESTS}

Experimental measurements were performed on an in-house small-scale test bench, as shown in Fig. 10 and Fig. 11.

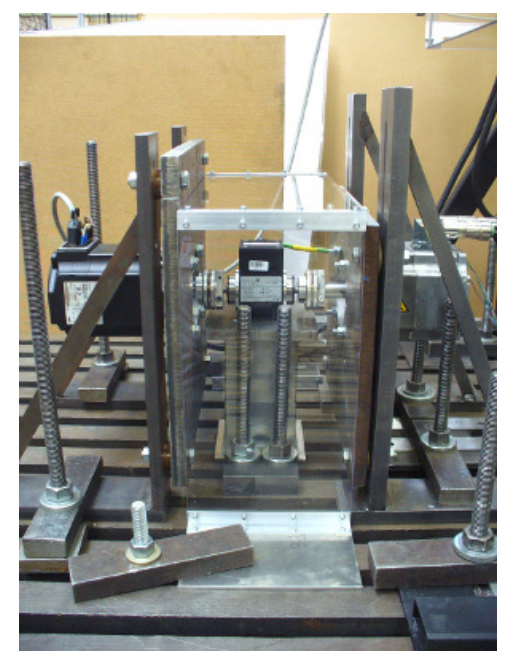

Fig. 10. Structure of the test-bench: the back-to-back motor configuration.

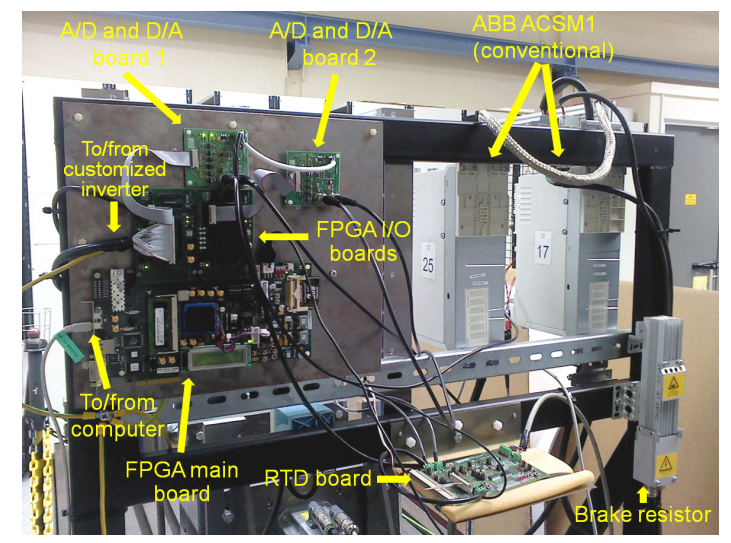

Fig. 11. Structure of the test-bench: the control board.

The test bench is composed by two permanent magnet synchronous machines in a back-to-back configuration, sharing a torquemeter on the shaft as shown in Fig. 10. The machine that emulates the wind turbine (on the left side) is a $1,92-\mathrm{kW}$ servomotor with a rated speed of $3000 \mathrm{rpm}$ and a rated torque of $6,1 \mathrm{Nm}$, while the machine acting as the generator (right side) is a 1,48-kW servomotor with a rated speed of $3000 \mathrm{rpm}$ and a rated torque of $4,7 \mathrm{Nm}$.

The machines are controlled by two 4-kW ABB ACSM1 converters. The load machine (i.e. wind turbine emulator) is connected to a conventional ACSM1 which performs closedloop speed and torque control through its own control board. The speed regulator parameters have been released accordingly to the considerations of Sect. II. The torque references representing the disturbances of the wind and the mechanical drive train (see Fig. 2) are added through an external analog signal, connected to one of the digital-to-analog (D/A) board shown in Fig. 11. At last, the D/A board is connected to the main control board, composed by a Xilinx Virtex 6 FPGA. All the models for the wind turbine emulation are implemented and executed in real-time in the FPGA environment.

The main machine emulating the generator is either controlled by a conventional ACSM1 in torque control mode, or through a customised inverter (behind the main control board of Fig. 11, not visible in the figure), which mounts a power unit from an ACSM1 and a custom interface for direct data exchange with the FPGA. In the latter case, the generator control is performed at lower levels, encompassing current/torque control in the FPGA. Such configuration allows more flexibility for control algorithm development.

Fig. 12 shows a typical speed profile of the running emulator. The speed is not constant but has a mean value of $200 \mathrm{rpm}$ determined by the low-bandwidth speed control, resembling the pitch system, with harmonics superimposed to it. A closer look at the speed (Fig. 13) reveals that the envelope is composed by third-order harmonics with random amplitude, according to the models in Sect. III.

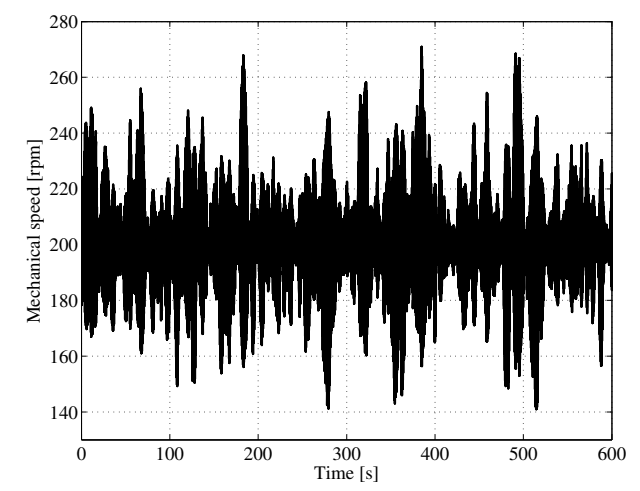

Fig. 12. Speed profile during normal operation of the wind turbine emulator.

Fig. 14 reports the experimental torque disturbances, similar to those shown in Fig. 6. At last, Fig. 15 and Fig. 16 show the reaction of the speed control to the disturbances. While not completely free of harmonics (that is, the speed PI regulation is somewhat trying to counterbalance the injected torque disturbances), the amplitude of the signal is lower than that of the injected pattern, thus resulting in the speed profile of Fig. 12. The harmonics are located around $10 \mathrm{~Hz}$, which is the third harmonic of the rotating frequency located around $200 \mathrm{rpm}(3,33 \mathrm{~Hz})$.

\section{CONCLUSIONS}

The paper presents a wind turbine emulator implemented on a small-scale test bench, with purposes of control algorithm 


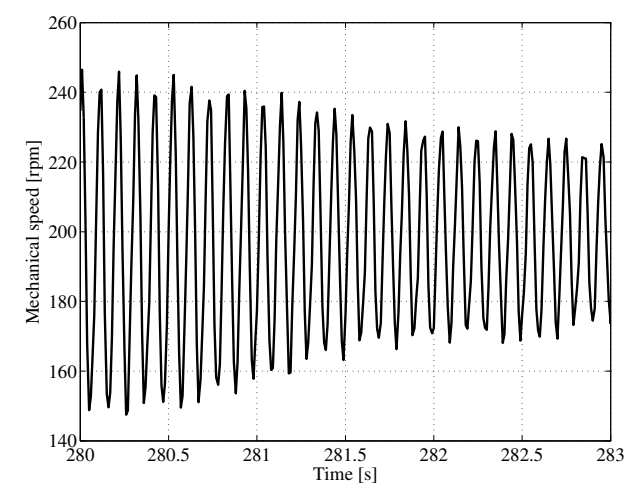

Fig. 13. Zoom of the speed profile.

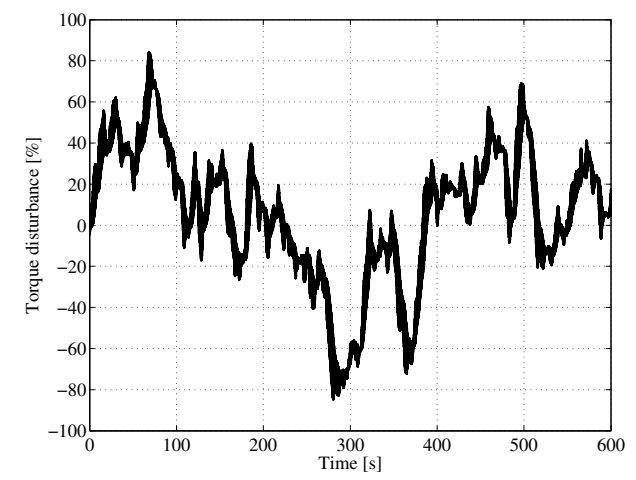

Fig. 14. Experimental torque disturbances injected in the cascade control.

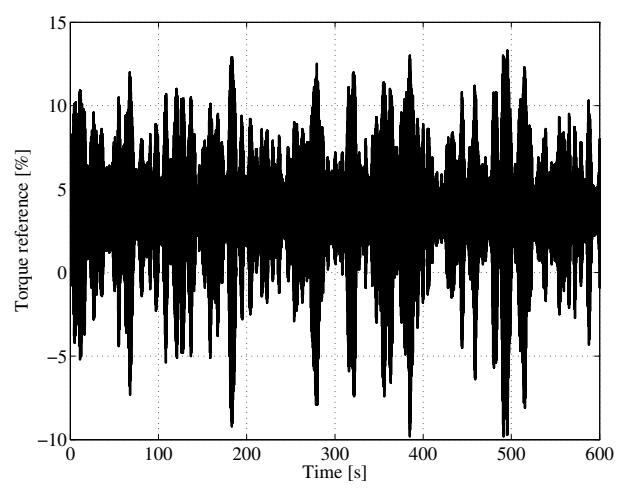

Fig. 15. Torque reference generated by the speed PI regulator.

development for the wind turbine generator. Smaller-scale test benches prove to be a useful tool for testing the control strategies before the actual development on the ABB ACS800 low-voltage converter family for wind power applications. An analysis of the required features of the emulator was performed, both in terms of models of the external factors (i.e. the wind) and of the mechanical characteristics of the wind turbine drive train. Implementation hints for FPGA-based control boards were given, proven by experimental results. It is also worth to note that a the performed analysis, given for multi-MW horizontal turbines, applies almost fully to small-scale domestic vertical turbines, but with a different mechanical behaviour.

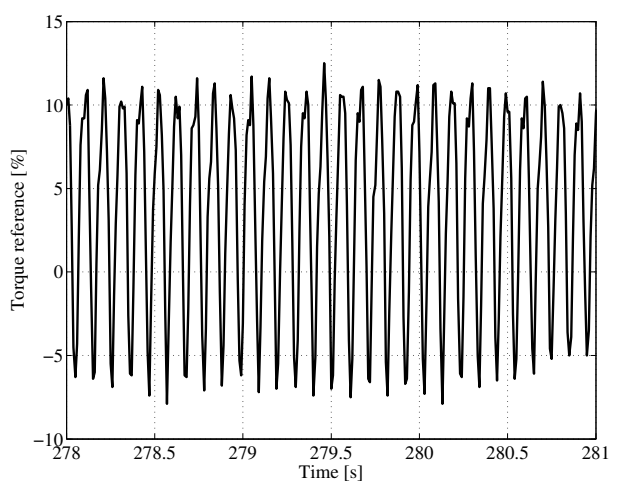

Fig. 16. Zoom on the torque reference generated by the speed PI regulator.

\section{REFERENCES}

[1] T. Thiringer and J.-Å. Dahlberg, "Periodic pulsations from a three-bladed wind turbine," IEEE Trans. Energy Convers., vol. 16, no. 2, Jun. 2001.

[2] J. G. Slootweg, S. W. H. de Haan, H. Polinder, and W. L. Kling, "General model for representing variable speed wind turbines in power system dynamics simulations," IEEE Trans. Power Syst., vol. 18, no. 1, pp. 144-151, 2003.

[3] P. Sørensen, N. A. Cutululis, A. Vigueras-Rodriguez, L. E. Jensen, J. Hjerrild, M. H. Donovan, and H. Madsen, "Power fluctuations from large wind farms," IEEE Trans. Power Syst., vol. 22, no. 3, pp. 958-965, Aug. 2007.

[4] P. Sørensen, N. A. Cutululis, A. Vigueras-Rodríguez, H. Madsen, P. Pinson, L. E. Jensen, J. Hjerrild, and M. Donovan, "Modelling of power fluctuations from large offshore wind farms," Wind Energ., no. 11, pp. 29-43, 2008

[5] H. M. Kojabadi, C. Liuchen, and T. Boutot, "Development of a novel wind turbine simulator for wind energy conversion systems using an inverter-controlled induction motor," IEEE Trans. Energy Convers., vol. 19, no. 3, pp. 547-552, Sep. 2004.

[6] S.-H. Song, B.-C. Jeong, H.-I. Lee, J.-J. Kim, J.-H. Oh, and G. Venkataramanan, "Emulation of output characteristics of rotor blades using a hardware-in-loop wind turbine simulator," in Proceedings of the Twentieth Annual IEEE Applied Power Electronics Conference and Exposition (APEC 2005), Austin, Texas, USA, Mar. 6-10 2005, pp. 1791-1796.

[7] B. Neammanee, S. Sirisumrannukul, and S. Chatratana, "Development of a wind turbine simulator for wind generator testing," International Energy Journal, no. 8, pp. 21-28, 2007.

[8] D. S. L. Dolan and P. W. Lehn, "Real-time wind turbine emulator suitable for power quality and dynamic control studies," in Proceedings of the International Conference on Power Systems Transients (IPST 2005), Montréal, Canada, Jun. 19-23 2005.

[9] - "Simulation model of wind turbine $3 p$ torque oscillations due to wind shear and tower shadow," IEEE Trans. Energy Convers., vol. 21, no. 3, Sep. 2006.

[10] ABB, "Technical Applications Papers No. 13 -Wind power plants," ABB SACE L.V. Breakers, Bergamo, Italy, Tech. Rep. 1SDC007112G020110/2011-4.000, Oct. 2011.

[11] G. Marsaglia. (1994, Aug. 1st,) Yet another RNG. [Online]. Available: https:/groups.google.com/forum/?fromgroups\#!topic/sci.stat.math/ p7aLW3TsJys[1-25]

[12] L. Peretti and V. Särkimäki, "Mechanical drive train emulation by means of electrical drives - a generalised approach," in Proceedings of the Industrial Electronics Society Conference (IECON) 2012, Montréal, Canada, Oct. 25-28, 2012, pp. 1878-1883.

[13] G. Bir and J. Jonkman, "Aeroelastic instabilities of large offshore and onshore wind turbines," National Renewable Energy Laboratory, Golden, Colorado, USA, Tech. Rep. NREL/CP-500-41804, Aug. 2007. [Online]. Available: http://www.nrel.gov/docs/fy07osti/41804.pdf 